Bryn Mawr College

Scholarship, Research, and Creative Work at Bryn Mawr

College

Physics Faculty Research and Scholarship

Physics

2017

\title{
Photoelectron angular distributions from rotationally resolved autoionizing states of $\mathrm{N} 2$
}

\author{
Alexander M. Chartrand \\ Elizabeth F. McCormack \\ Ugo Jacovella \\ ETH Zurich \\ David M P Holland \\ Daresbury Laboratory \\ Berenger Gans \\ Université Paris-Saclay \\ See next page for additional authors
}

Bryn Mawr College, achartrand@brynmawr.edu

Bryn Mawr College, emccorma@brynmawr.edu

Let us know how access to this document benefits you.

Follow this and additional works at: http://repository.brynmawr.edu/physics_pubs

Part of the Atomic, Molecular and Optical Physics Commons

\section{Citation}

Chartrand, Alexander M.; McCormack, Elizabeth F.; Jacovella, Ugo; Holland, David M P; Gans, Berenger; Tang, Xiaofeng; García, Gustavo A.; Nahon, Laurent; and Pratt, Stephen T., "Photoelectron angular distributions from rotationally resolved autoionizing states of N2" (2017). Physics Faculty Research and Scholarship. 111.

http://repository.brynmawr.edu/physics_pubs/111

This paper is posted at Scholarship, Research, and Creative Work at Bryn Mawr College. http://repository.brynmawr.edu/physics_pubs/111

For more information, please contact repository@brynmawr.edu. 
Authors

Alexander M. Chartrand, Elizabeth F. McCormack, Ugo Jacovella, David M P Holland, Berenger Gans, Xiaofeng Tang, Gustavo A. García, Laurent Nahon, and Stephen T. Pratt 
Photoelectron angular distributions from rotationally resolved autoionizing states of $\mathrm{N}_{2}$

A. M. Chartrand, E. F. McCormack, U. Jacovella, D. M. P. Holland, B. Gans, Xiaofeng Tang, G. A. Garcia, L. Nahon, and S. T. Pratt

Citation: The Journal of Chemical Physics 147, 224303 (2017);

View online: https://doi.org/10.1063/1.5004538

View Table of Contents: http://aip.scitation.org/toc/jcp/147/22

Published by the American Institute of Physics

AIP $\mid$ The Journal of

Chemical Physics

PERSPECTIVES 


\title{
Photoelectron angular distributions from rotationally resolved autoionizing states of $\mathbf{N}_{\mathbf{2}}$
}

\author{
A. M. Chartrand, ${ }^{1}$ E. F. McCormack, ${ }^{1, a)}$ U. Jacovella, ${ }^{2}$ D. M. P. Holland,${ }^{3}$ B. Gans, ${ }^{4}$ \\ Xiaofeng Tang, ${ }^{5, b)}$ G. A. Garcia, ${ }^{5}$ L. Nahon, ${ }^{5}$ and S. T. Pratt ${ }^{6}$ \\ ${ }^{1}$ Department of Physics, Bryn Mawr College, Bryn Mawr, Pennsylvania 19010, USA \\ ${ }^{2}$ Laboratorium für Physikalische Chemie, ETH Zürich, 8093 Zürich, Switzerland \\ ${ }^{3}$ STFC, Daresbury Laboratory, Daresbury, Warrington, Cheshire WA4 4AD, United Kingdom \\ ${ }^{4}$ Institut des Sciences Moléculaires d'Orsay (ISMO), UMR 8214 CNRS, Univ. Paris-Sud, \\ Université Paris-Saclay, F-91405 Orsay, France \\ ${ }^{5}$ Synchrotron Soleil, L'Orme des Merisiers, F-91192 Gif-sur-Yvette, France \\ ${ }^{6}$ Argonne National Laboratory, Argonne, Illinois 60439, USA
}

(Received 13 September 2017; accepted 13 November 2017; published online 8 December 2017)

\begin{abstract}
The single-photon, photoelectron-photoion coincidence spectrum of $\mathrm{N}_{2}$ has been recorded at high $\left(\sim 1.5 \mathrm{~cm}^{-1}\right)$ resolution in the region between the $\mathrm{N}_{2}{ }^{+} X^{2} \Sigma_{\mathrm{g}}{ }^{+}, v^{+}=0$ and 1 ionization thresholds by using a double-imaging spectrometer and intense vacuum-ultraviolet light from the Synchrotron SOLEIL. This approach provides the relative photoionization cross section, the photoelectron energy distribution, and the photoelectron angular distribution as a function of photon energy. The region of interest contains autoionizing valence states, vibrationally autoionizing Rydberg states converging to vibrationally excited levels of the $\mathrm{N}_{2}{ }^{+} X^{2} \Sigma_{\mathrm{g}}{ }^{+}$ground state, and electronically autoionizing states converging to the $\mathrm{N}_{2}{ }^{+} A^{2} \Pi$ and $B^{2} \Sigma_{\mathrm{u}}{ }^{+}$states. The wavelength resolution is sufficient to resolve rotational structure in the autoionizing states, but the electron energy resolution is insufficient to resolve rotational structure in the photoion spectrum. A simplified approach based on multichannel quantum defect theory is used to predict the photoelectron angular distribution parameters, $\beta$, and the results are in reasonably good agreement with experiment. Published by AIP Publishing. https://doi.org/10.1063/1.5004538
\end{abstract}

\section{INTRODUCTION}

The near-threshold, single-photon ionization spectrum of molecular nitrogen shows a relatively weak direct ionization continuum along with significant intense autoionization features that can be either sharp or quite broad. ${ }^{1-7}$ Between the ionization threshold ${ }^{8}$ and $\sim 127850 \mathrm{~cm}^{-1}$, autoionization is energetically constrained to leave the ion in the $\mathrm{N}_{2}{ }^{+} X^{2} \Sigma_{\mathrm{g}}{ }^{+}$, $v^{+}=0$ state. In this region, autoionization features arise from several different sources: autoionization of Rydberg series converging to vibrationally excited levels of the $\mathrm{N}_{2}{ }^{+} X^{2} \Sigma_{\mathrm{g}}{ }^{+}$ ground state; autoionization of Rydberg series converging to electronically excited states of $\mathrm{N}_{2}{ }^{+}$(notably the $A^{2} \Pi_{\mathrm{u}}$ and $B^{2} \Sigma_{\mathrm{u}}{ }^{+}$states); and autoionization of high vibrational levels of the $b^{\prime}{ }^{1} \Sigma_{\mathrm{u}}{ }^{+}$and $b^{1} \Pi_{\mathrm{u}}$ valence states. ${ }^{1-7}$ This relatively large number of states can lead to complex resonance features that are perturbed with respect to intensities, linewidths, and expected level positions. Thus, while considerable progress has been made in the analysis of this region of the $\mathrm{N}_{2}$ spectrum, ${ }^{1-18}$ some strong features still lack definitive spectral assignments.

\footnotetext{
a)Present address: Department of Physics and Astronomy, Bowdoin College, Brunswick, Maine 04011, USA.

b)Present address: Anhui Institute of Optics and Fine Mechanics, Chinese Acadmey of Sciences, Hefei 230031, Anhui, China.
}

Perhaps the most noteworthy of these regions lies between $\sim 126200$ and $126500 \mathrm{~cm}^{-1}$ in the single-photon ionization spectrum. ${ }^{1,5-7,14-17}$ This region contains a number of sharp bands along with two intense, broad-yet-structured, complex resonances that have been said to have the appearance of two towers of a gothic cathedral. There is substantial agreement over the assignment of most of the sharp structure, and the lower energy tower has been assigned to a transition associated with the $\left(B^{2} \Sigma_{\mathrm{u}}{ }^{+}\right) 3 s \sigma, v^{\prime}=0$ final state; however, despite numerous theoretical ${ }^{4,9,14,15}$ and experimental studies, no consensus has been reached for the assignment of the higher energy tower. These previous experiments include extremely high-resolution single-photon absorption and ionization of jetcooled $\mathrm{N}_{2},{ }^{5,16}$ photoionization from vibrationally excited levels of the neutral electronic ground state, ${ }^{1}$ double-resonance studies via the $a^{1} \Pi_{\mathrm{g}}$ state, ${ }^{10-12}$ and comprehensive studies using isotopic substitution. ${ }^{17}$

In principle, photoelectron angular distributions can provide insight into the photoionization dynamics and the electronic character of the autoionizing resonances and ejected photoelectron. ${ }^{6,719-25}$ Such studies are most informative when the excitation process is rotationally resolved and when the final ionic state is vibrationally and rotationally resolved. ${ }^{26-30}$ There have been a number of previous studies of the photoelectron angular distributions of $\mathrm{N}_{2}$ just above the first ionization threshold. In recent studies by O'Keeffe et al. ${ }^{6}$ and Holland et al.,$^{7}$ velocity map imaging was used to record photoelectron 
energy and angular distributions as a function of wavelength between threshold and $\sim 135000 \mathrm{~cm}^{-1}$. In this region, the $\mathrm{N}_{2}{ }^{+}$ion can be left in the $X^{2} \Sigma_{\mathrm{g}}{ }^{+}$state with $v^{+} \leq 4$, and the electron energy resolution ${ }^{6,7}$ was sufficient to resolve vibrational, but (for the most part) not rotational, levels of the ion. The photon resolution in the two studies ${ }^{6,7}$ was comparable and $\sim 7-10 \mathrm{~cm}^{-1}$, and, as a result, the rotational structure in the excitation process was not resolved. The two studies gave qualitatively similar results for the vibrational branching ratios and photoelectron angular distributions, but some significant quantitative differences were noted at low electron energies. $^{7}$

In this paper, we present new results on the near-threshold photoionization of molecular nitrogen by making use of the high-resolution vacuum-ultraviolet (VUV) light provided at the DESIRS end station of the Synchrotron SOLEIL ${ }^{31}$ and the DELICIOUS III imaging photoelectron-photoion coincidence spectrometer $^{32,33}$ that allows the recording of electron energy and angular distributions across the region of interest. While the electron spectrometer resolution is not sufficient to resolve the final $\mathrm{N}_{2}{ }^{+} X^{2} \Sigma_{\mathrm{g}}{ }^{+}, v^{+}=0$ rotational distributions, the photon resolution is sufficient to resolve rotational structure in the excitation process for most of the observed bands. Figure 1 shows the experimental photoionization spectrum across the full region of interest, along with the corresponding angular distribution parameter, $\beta$. Rotational resolution in the excitation process allows a significant simplification in the analysis of the photoelectron angular distributions. In particular, as shown in Sec. III, we can predict the photoelectron angular distributions for a variety of rotationally resolved autoionizing resonances in $\mathrm{N}_{2}$ by using a simplification of the multichannel quantum defect theory (MQDT) machinery ${ }^{26,34,35}$ originally developed for $\mathrm{H}_{2}$. The predictions are in reasonable agreement with the present experimental results and provide insight into the assignments of the autoionizing resonances as well as into the corresponding decay mechanisms.

In what follows, we first describe the experimental approach, followed by the theoretical approach. We then

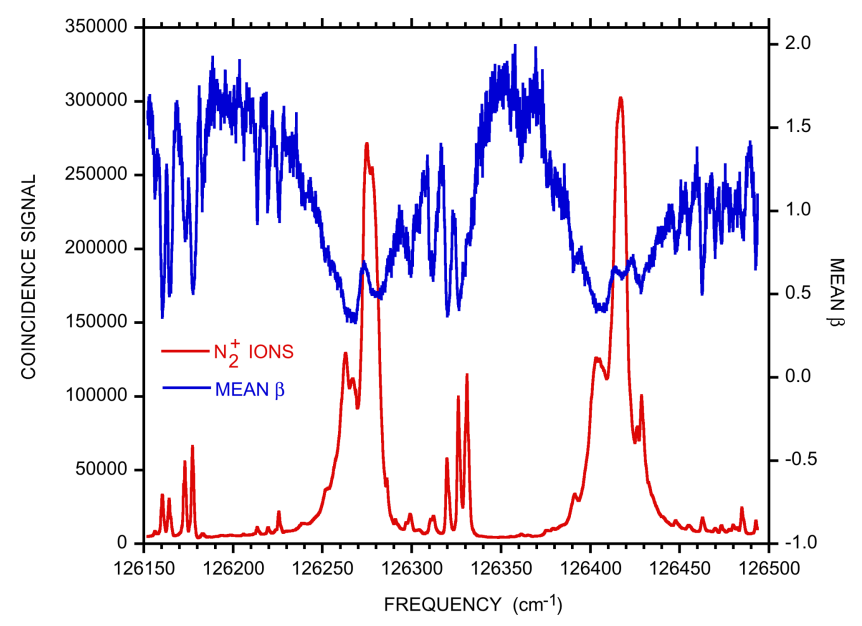

FIG. 1. The photoionization spectrum of $\mathrm{N}_{2}$ in the region just above the first ionization threshold to produce $\mathrm{N}_{2}{ }^{+} X^{2} \Sigma_{\mathrm{g}}{ }^{+}, v^{+}=0$, along with the photoelectron angular distribution parameter, $\beta$. In this region, only the lowest vibrational level of the ion is energetically accessible, and the rotational structure in the ion is not resolved. See text for details. discuss the experimental results and the comparison with theoretical predictions and conclude with a brief discussion of the implications of this study.

\section{EXPERIMENT}

The experiments were performed by using the DELICIOUS III double-imaging photoelectron-photoion coincidence spectrometer on the DESIRS undulator-based beamline at Synchrotron SOLEIL. The apparatus and beamline have been described in detail previously. ${ }^{31-33}$ For the present experiments, pure $\mathrm{N}_{2}$ at 2 bars backing pressure was expanded through a $70 \mu \mathrm{m}$-diameter nozzle, and the resulting continuous molecular beam was collimated by two $1 \mathrm{~mm}$ skimmers before passing into the interaction region of the spectrometer. The measured velocity spread in the molecular beam as determined by ion imaging yields a translational temperature of $20 \mathrm{~K}$. The observed rotational band envelopes are consistent with this temperature, although they may also be affected by competing heterogeneous predissociation processes. For the most part, the observed transitions are limited to those from $\mathrm{N}_{2} X^{1} \Sigma_{\mathrm{g}}{ }^{+}, v^{\prime \prime}=0$ levels with $J^{\prime \prime} \leq 3$. The linearly polarized VUV light from the undulator was monochromatized by the $6.65 \mathrm{~m}$ normal-incidence monochromator equipped for this study with a 2400 grooves/mm grating. The resulting VUV beam crossed at right angle to both the molecular beam axis and the spectrometer axis. Wavelength scans were recorded with a step size of $0.2 \mathrm{~cm}^{-1}$, and the sharpest features in the spectrum show full width at halfmaximum (FWHM) linewidths of $\sim 1.5 \mathrm{~cm}^{-1}$. The energy scale was calibrated by using several previously reported rotational lines in this region ${ }^{5,8}$ and is expected to be accurate to $\sim \pm 1-2 \mathrm{~cm}^{-1}$.

The photoelectron-photoion coincidence data were processed to yield the photoelectron images coincident with the mass $28, \mathrm{~N}_{2}{ }^{+}$ion signal. These images were reconstructed by using the pBasex algorithm ${ }^{36}$ to give both the photoelectron energy distribution and angular distribution as a function of wavelength. Because the photon energy lies below the $\mathrm{N}_{2}{ }^{+} X^{2} \Sigma_{\mathrm{g}}{ }^{+}, v^{+}=1$ threshold, and because the $\mathrm{N}_{2}{ }^{+} X^{2} \Sigma_{\mathrm{g}}{ }^{+}$, $v^{+}=0$ rotational levels are not resolved, the photoelectron energy distributions show a single peak corresponding to the production of the $v^{+}=0$ ions.

The photoelectron angular distribution for single-photon ionization with linearly polarized light must have the form ${ }^{26,34}$

$$
\frac{d \sigma}{d \Omega}=\frac{\sigma}{4 \pi}\left[1+\beta P_{2}(\cos \theta)\right],
$$

where $\sigma$ is the photoionization cross section, $d \Omega$ defines the solid angle element, $\theta$ is the angle between the polarization axis and the electron velocity vector, and $P_{2}(\cos \theta)$ is the Legendre polynomial $\left(3 \cos ^{2} \theta-1\right) / 2$. At each wavelength, the pBasex reconstruction provides $\beta$ as a function of electron kinetic energy, and this value is typically averaged across the finite kinetic energy range of the photoelectron peak. Small differences in the $\beta$ values arise depending on the range of kinetic energies used, for example, averaging over the full width at half-maximum of the peak yields slightly different $\beta$ values than averaging over the full width at one-tenth of the 
maximum. In what follows, we have averaged the beta results of these two approaches.

\section{THEORETICAL ANGULAR DISTRIBUTION PARAMETERS}

Here we develop theoretical expressions for the photoelectron angular distributions of $\mathrm{N}_{2}$ based on the MQDT framework of Dill and Fano ${ }^{34,35}$ and as implemented by Raoult et al. ${ }^{26}$ for the photoionization of molecular hydrogen. The application to $\mathrm{N}_{2}$ is straightforward due to the happy coincidence that the ground state neutral and ion have the same symmetry as in $\mathrm{H}_{2}$, i.e., ${ }^{1} \Sigma_{\mathrm{g}}{ }^{+}$and ${ }^{2} \Sigma_{\mathrm{g}}{ }^{+}$symmetry, respectively, and thus single-photon ionization near threshold leads to the ejection of an odd- $\ell$ photoelectron. $\mathrm{In}_{2}$, the single-photon dynamics are dominated by $\ell=1(p)$ electrons as the $\ell=3(f)$ electrons are essentially non-penetrating. In $\mathrm{N}_{2}$, the $f \sigma$ and $f \pi$ components of the $f$ states are more penetrating, but experiments still show that the $n p$ Rydberg series are much stronger than the corresponding $n f$ series. ${ }^{4,9}$ Indeed, the bands associated with transitions to $n f$ final states are strongest when they gain intensity through perturbations by Rydberg states converging to the $A^{2} \Pi_{\mathrm{u}}$ ionic state. ${ }^{4}$ Thus, near threshold, direct photoionization in $\mathrm{N}_{2}$ should be dominated by the $p$ continua.

\section{A. Direct ionization into the open continuum}

In the absence of autoionizing resonances, direct photoionization into the continuum leads to a relatively simple expression if the photoelectron corresponds to a pure $p$ wave. In particular, if the final rotational state is unresolved, Dill ${ }^{34}$ has shown that the angular distribution parameter, $\beta_{p}$, is given by

$$
\beta_{p}=\frac{2\left(2 d_{\sigma}^{2}+7 d_{\pi}^{2}+6 d_{\sigma} d_{\pi} \cos \pi \delta\right)}{5\left(d_{\sigma}^{2}+2 d_{\pi}^{2}\right)},
$$

where $d_{\sigma}$ and $d_{\pi}$ are the transition moments to eject $p \sigma$ and $p \pi$ photoelectrons, respectively, and the phase-shift difference is $\delta=\mu_{\sigma}-\mu_{\pi}$, where $\mu_{\lambda}$ is the threshold quantum defect. The relevant transition moments and quantum defect parameters have been reported previously in two publications: first by Raoult et al. ${ }^{18}$ and later by Jungen et al. ${ }^{4}$ The two sets of parameters yield direct photoionization $\beta_{p}$ values of 0.415 and 1.973 , respectively. While the magnitudes of the quantum defect parameters and transition moments are similar in the two studies, $d_{\sigma}$ and $d_{\pi}$ have opposite signs in the former study ${ }^{18}$ and the same signs in the latter study, ${ }^{4}$ which results in the substantially different $\beta_{p}$ values. The results presented below appear to support the results of Jungen et al.

\section{B. Autoionization of $\left(X^{2} \Sigma_{g}{ }^{+}\right) 6 p \sigma_{u}, v=2$ and other ${ }^{1} \Sigma_{\mathrm{u}}{ }^{+}$states}

Figure 1 shows a number of sharp, rotationally resolved bands that have been assigned previously, and we now focus on developing expressions for $\beta$ values for these bands. The sharp band between 126150 and $126190 \mathrm{~cm}^{-1}$ corresponds to the transition to the $\left(X^{2} \Sigma_{\mathrm{g}}{ }^{+}\right) 6 p \sigma_{u}, v^{\prime}=2$ state. ${ }^{5,10}$ The final state of this band is sufficiently low in principal quantum number, $n$, such that we assume the projection of the orbital angular momentum of the Rydberg electron on the internuclear axis, $\lambda$, is a good quantum number, as is the projection of the total angular momentum, $\Lambda$. This state undergoes vibrational autoionization by converting two quanta of vibrational energy into electronic (translational) energy of the escaping electron. Because the ion has ${ }^{2} \Sigma_{\mathrm{g}}{ }^{+}$symmetry and the $p \sigma$ autoionizing resonance has ${ }^{1} \Sigma_{\mathrm{u}}{ }^{+}$symmetry, the photoelectron must have odd $\ell$ and $\sigma_{u}$ symmetry. Because $p-f$ mixing is relatively weak ${ }^{4}$ in $\mathrm{N}_{2}$, the dominant process will result in a $p \sigma_{u}$ photoelectron, with the ion left in an $X^{2} \Sigma_{\mathrm{g}}{ }^{+}$, $v^{+}=0, N^{+}$rotational level consistent with the conservation of angular momentum. We note that Park et al. observed substantially more $\ell$ mixing in the vibrational autoionization of an $n s$ Rydberg state of $\mathrm{NO}^{29}$ However, in contrast to the weak $p-f$ mixing in $\mathrm{N}_{2}$, the $s-d$ mixing in NO is quite strong, and because $\mathrm{NO}$ is a heteronuclear molecule, even-odd $\ell$ mixing is also possible.

For the band corresponding to the transition to the $\left(X^{2} \Sigma_{\mathrm{g}}{ }^{+}\right) 6 p \sigma, v^{\prime}=2$ state, the excitation process is rotationally resolved, and we seek the expression for the angular distribution for the process,

$$
\begin{aligned}
& \mathrm{N}_{2} X^{1} \Sigma_{\mathrm{g}}{ }^{+}, v^{\prime \prime}=0, J^{\prime \prime} \rightarrow\left(X^{2} \Sigma_{\mathrm{g}}{ }^{+}\right) 6 p \sigma_{u}, v^{\prime}=2, J^{\prime} \\
& \rightarrow \mathrm{N}_{2}{ }^{+} X^{2} \Sigma_{\mathrm{g}}{ }^{+}, v^{+}=0, N^{+}+p \sigma_{u},
\end{aligned}
$$

averaged over all of the allowed $N^{+}$rotational levels.

Dill ${ }^{34}$ has used the angular momentum transfer, $j_{t}$, formalism of Fano and Dill ${ }^{35}$ to derive an expression for the angular distribution parameter for photoionization from a single $J^{\prime \prime}$ level of an $X^{1} \Sigma_{\mathrm{g}}{ }^{+}$state into the continuum of a single $N^{+}$ level of a ${ }^{2} \Sigma_{g}{ }^{+}$ion with the ejection of an $\ell=1$ photoelectron. As given by Raoult et al. ${ }^{26}$ this expression is

$$
\beta_{v^{+} N^{+} \leftarrow v^{\prime \prime} J^{\prime \prime}}=\frac{2\left|\left\langle v^{+} N^{+}|\bar{S}(0)| v^{\prime \prime} J^{\prime \prime}\right\rangle\right|^{2}-3\left|\left\langle v^{+} N^{+}|\bar{S}(1)| v^{\prime \prime} J^{\prime \prime}\right\rangle\right|^{2}+\left|\left\langle v^{+} N^{+}|\bar{S}(2)| v^{\prime \prime} J^{\prime \prime}\right\rangle\right|^{2}}{\left|\left\langle v^{+} N^{+}|\bar{S}(0)| v^{\prime \prime} J^{\prime \prime}\right\rangle\right|^{2}+3\left|\left\langle v^{+} N^{+}|\bar{S}(1)| v^{\prime \prime} J^{\prime \prime}\right\rangle\right|^{2}+5\left|\left\langle v^{+} N^{+}|\bar{S}(2)| v^{\prime \prime} J^{\prime \prime}\right\rangle\right|^{2}} .
$$

Here, $\bar{S}\left(j_{t}\right)$ are rotationally invariant amplitudes related to the scattering matrix, $S(J),{ }^{26}$

$$
\left\langle v^{+} N^{+}\left|\bar{S}\left(j_{t}\right)\right| v^{\prime \prime} J^{\prime \prime}\right\rangle=\sum_{J}(-1)^{J-J^{\prime \prime}+1}(2 J+1)\left\{\begin{array}{ccc}
1 & 1 & j_{t} \\
J^{\prime \prime} & N^{+} J
\end{array}\right\}\left\langle v^{+} N^{+}|S(J)| v^{\prime \prime} J^{\prime \prime}\right\rangle .
$$

Here, $J$ is the total angular momentum of the final state photoelectron and ion, which for an autoionizing state is equal to $J^{\prime}$, and the angular momentum transfer, $j_{t}$, is given by ${ }^{35}$ 


$$
j_{t}=\vec{N}^{+}-\vec{J}^{\prime \prime}=\vec{j}_{r}-\vec{\ell} \quad \text { and } \quad \vec{j}_{r}=1,
$$

where $\vec{j}_{r}$ is the angular momentum of the photon and the $\bar{S}\left(j_{t}\right)$ terms in Eq. (5) are restricted to those with allowed values of $j_{t}$. For $\ell=1, j_{t}$ is restricted to 0,1 , or 2 .

In general, the total angular momentum following photoabsorption, $J^{\prime}=J^{\prime \prime}, J^{\prime \prime} \pm 1$, and the sum in Eq. (5) requires three $S(J)$ values. However, each autoionizing resonance corresponds to a single value of $J^{\prime}$, which is preserved upon autoionization. If the resonance is strong relative to the continuum, we can limit our considerations to this single value of $J=J^{\prime}$. (An approximate approach to take the continuum into account is discussed in Sec. IV.) Thus, we have a single value of $S(J)$ that can be taken as a constant and ultimately factored out of the expression for $\beta$ in Eq. (4). With these approximations, we can evaluate Eq. (4) to determine values for $\beta_{v^{+} N^{+} \leftarrow v^{\prime \prime} N^{\prime \prime}}(J)$.

As discussed above, although we do not resolve individual $N^{+}$levels, we have made the assumption that the photoelectron has $p \sigma$ symmetry and that the final total symmetry is ${ }^{1} \Sigma_{\mathrm{u}}{ }^{+}$. Thus, we can simply use the geometric frame transformation elements, ${ }^{26}\left\langle N^{+} \mid \Lambda^{+}\right\rangle^{J}$, to project this channel onto the allowed $N^{+}$states. With this approximation, the rotationally averaged $\beta$ value becomes

$$
\beta_{v^{+} \bar{N}^{+} \leftarrow v^{\prime \prime} J^{\prime \prime}}=\sum_{N^{+}}\left\langle N^{+} \mid \Lambda\right\rangle^{J} \beta_{v^{+} N^{+} \leftarrow v^{\prime \prime} J^{\prime \prime}},
$$

where $\bar{N}^{+}$indicates a sum over allowed $N^{+}$levels. The resulting values are compared with the experiment in Sec. IV.

Figure 1 also includes bands corresponding to transitions to the $b^{\prime}{ }^{1} \Sigma_{\mathrm{u}}{ }^{+}, v^{\prime}=42-44$ states, the $\left(B^{2} \Sigma_{\mathrm{u}}{ }^{+}\right) 3 s \sigma_{g}, v^{\prime}=0$ state, an unassigned ${ }^{1} \Sigma_{\mathrm{u}}{ }^{+}$or ${ }^{1} \Pi_{\mathrm{u}}$ state most likely having an $A^{2} \Pi_{\mathrm{u}}$ core, and the $\left(X^{2} \Sigma_{\mathrm{g}}{ }^{+}\right) 9 f, v^{\prime}=1$ state. ${ }^{4-12}$ The latter is best described in Hund's case (d) ${ }^{12,13}$ and is discussed in more detail later on. All of the former bands can be treated at least tentatively in Hund's case (a) or (b). If the autoionizing resonances are much stronger than the continuum, we can still assign $J^{\prime \prime}$ and $J^{\prime}$. If $\Lambda^{\prime}$ of the autoionizing resonances is known, and the final state is $X^{2} \Sigma_{\mathrm{g}}{ }^{+}, v^{+}=0$, the nominal $\lambda$ value of the photoelectron is also known and $\ell$ must be odd. In general, $\ell$ will likely be limited to 1 or 3 because higher values will be non-penetrating. The balance between $\ell=1$ and 3 will be determined by the character of the autoionizing resonance and the autoionizing mechanism.

The $b^{\prime}{ }^{1} \Sigma_{\mathrm{u}}{ }^{+}$valence state has a mixed configuration, ${ }^{4,37}$ with the two dominant contributions coming from $\ldots\left(1 \pi_{u}\right)^{3}\left(3 \sigma_{g}\right)^{2}\left(1 \pi_{g}\right)^{1}$ and $\ldots\left(1 \pi_{u}\right)^{4}\left(3 \sigma_{g}\right)^{1}\left(3 \sigma_{u}\right)^{1}$. The $1 \pi_{g}$ orbital of the former configuration has the characteristics of a $3 d \pi$ orbital, ${ }^{4}$ and the remaining part of the configuration corresponds to $\mathrm{N}_{2}{ }^{+} A^{2} \Pi_{\mathrm{u}}$. In the analysis of Jungen et al., ${ }^{4}$ the $s-p$ and $d-p$ interactions are larger than the $s-f$ and $d-f$ interactions, so we assume that the dominant decay is to the $X^{2} \Sigma_{\mathrm{g}}{ }^{+}$, $v^{+}=0+\varepsilon p \sigma_{u}$ continuum. The $\ldots\left(1 \pi_{u}\right)^{4}\left(3 \sigma_{g}\right)^{1}\left(3 \sigma_{u}\right)^{1}$ configuration corresponds to an $X^{2} \Sigma_{\mathrm{g}}{ }^{+}$core with a $3 \sigma_{u}$ excited electron. The $3 \sigma_{u}$ orbital has mixed $p \sigma_{u}$ and $f \sigma_{u}$ character. Because the $p \sigma_{u}$ orbital is more penetrating and known to be promoted, we make the simplifying approximation that it dominates the decay, once again resulting in $X^{2} \Sigma_{\mathrm{g}}{ }^{+}$, $v^{+}=0+\varepsilon p \sigma_{u}$.

For the $\left(B^{2} \Sigma_{\mathrm{u}}{ }^{+}\right) 3 s \sigma_{g}$ Rydberg state, autoionization is again expected to favor the $X^{2} \Sigma_{\mathrm{g}}{ }^{+}, v^{+}=0+\varepsilon p \sigma_{u}$ continuum over the $X^{2} \Sigma_{\mathrm{g}}{ }^{+}, v^{+}=0+\varepsilon f \sigma_{u}$ continuum due to stronger $s-p$ mixing. Similarly, autoionization of $\left(A^{2} \Pi_{\mathrm{u}}\right) 3 d \sigma_{g} / 4 s \sigma_{g}$ ${ }^{1} \Pi_{\mathrm{u}}$ Rydberg states is expected to favor the $X^{2} \Sigma_{\mathrm{g}}{ }^{+}, v^{+}=0+$ $\varepsilon p \pi_{u}$, or $\varepsilon f \pi_{u}$, or $\varepsilon p \pi_{u}$ continua for the $3 d \sigma_{g}$ and $4 s \sigma_{g}$ states, respectively. With the assumption that the $p$ continua dominate in all cases, we can thus use the same approach as for the $\left(X^{2} \Sigma_{\mathrm{g}}{ }^{+}\right) 6 p \sigma, v^{\prime}=2$ states, with the substitution that $\Lambda=1$ in Eq. (7) for the ${ }^{1} \Pi_{\mathrm{u}}$ autoionizing states. Somewhat remarkably, with these approximations, the $\beta$ values for vibrational autoionization of the $\left(X^{2} \Sigma_{\mathrm{g}}{ }^{+}\right) 6 p \sigma, v^{\prime}=2$ state, electronic autoionization of the $b^{\prime}{ }^{1} \Sigma_{\mathrm{u}}{ }^{+}$valence state, and electronic autoionization of the $\left(B^{2} \Sigma_{\mathrm{u}}{ }^{+}\right) 3 s \sigma_{g}{ }^{1} \Sigma_{\mathrm{u}}{ }^{+}$Rydberg state all have the same rotational dependence.

\section{Autoionization of $\left(X^{2} \Sigma_{g}{ }^{+}\right) 9 f, v=1$}

The formalism of Dill ${ }^{34}$ can be adapted for autoionization with the ejection of an $\ell=3$ photoelectron. With all else the same, $j_{t}=2,3$, or 4 , and the corresponding expression for the angular distribution parameter is

$$
\beta_{v^{+} N^{+} \leftarrow v^{\prime \prime} J^{\prime \prime}}=\frac{4\left|\left\langle v^{+} N^{+}|\bar{S}(2)| v^{\prime \prime} J^{\prime \prime}\right\rangle\right|^{2}-7\left|\left\langle v^{+} N^{+}|\bar{S}(3)| v^{\prime \prime} J^{\prime \prime}\right\rangle\right|^{2}+3\left|\left\langle v^{+} N^{+}|\bar{S}(4)| v^{\prime \prime} J^{\prime \prime}\right\rangle\right|^{2}}{5\left|\left\langle v^{+} N^{+}|\bar{S}(2)| v^{\prime \prime} J^{\prime \prime}\right\rangle\right|^{2}+7\left|\left\langle v^{+} N^{+}|\bar{S}(3)| v^{\prime \prime} J^{\prime \prime}\right\rangle\right|^{2}+9\left|\left\langle v^{+} N^{+}|\bar{S}(4)| v^{\prime \prime} J^{\prime \prime}\right\rangle\right|^{2}},
$$

with $\bar{S}\left(j_{t}\right)$ given by

$$
\begin{aligned}
\left\langle v^{+} N^{+}\left|\bar{S}\left(j_{t}\right)\right| v^{\prime \prime} J^{\prime \prime}\right\rangle= & \sum_{J}(-1)^{J-J^{\prime \prime}+1}(2 J+1)\left\{\begin{array}{ccc}
3 & 1 & j_{t} \\
J^{\prime \prime} & N^{+} & J
\end{array}\right\} \\
& \times\left\langle v^{+} N^{+}|S(J)| v^{\prime \prime} J^{\prime \prime}\right\rangle .
\end{aligned}
$$

The $9 f, v^{\prime}=1$ Rydberg state is best described in Hund's case (d), for which $\lambda$ and $\Lambda$ are not good quantum numbers. ${ }^{12}$ Thus, Eq. (7) will not adequately describe the rotational branching fractions and rotationally averaged $\beta$ values for autoionization of this state. Within the MQDT framework, the vibrational autoionization process can be viewed as a vibrationally inelastic collision of the Rydberg electron with the $v^{\prime}=1$ ion core and subsequent escape of the free electron into the $v^{+}=0$ continuum. At short range, the $\lambda$ and $\Lambda$ quantum numbers become good, and a frame transformation can be used to connect the long- and short-range regions. Assuming $\ell$ does not change, the matrix elements describing the $N^{+}$branching fractions are 


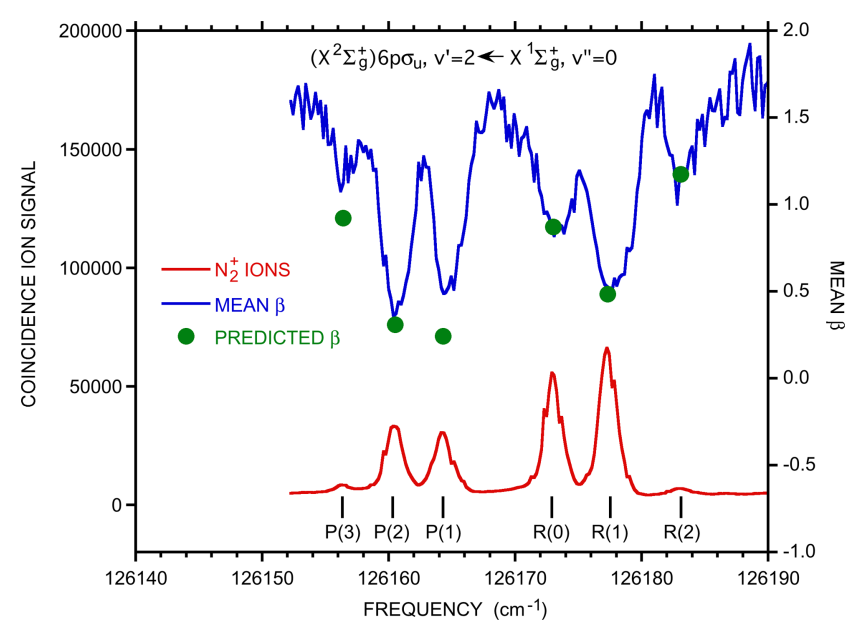

FIG. 2. Expanded portion of Fig. 1 in the region of the $\left(X^{2} \Sigma_{\mathrm{g}}{ }^{+}\right) 6 p \sigma$, $v^{\prime}=2$ band, along with the corresponding $\beta$ values. The theoretical $\beta$ values are the predicted values for the resonances corrected for the experimental direct photoionization continuum $\beta$ value, as discussed in the text. The $\mathrm{P}(3)$ transition is not listed in Table I because only one wavelength scan reached this feature.

described by

$$
\begin{aligned}
\left\langle\mathrm{N}_{2}^{+}, v^{+}\right. & \left.=0, N^{+}+\varepsilon f\left|S^{J}\right| \mathrm{N}_{2}, 9 f, v^{\prime}=1, R^{\prime}\right\rangle \\
& =\sum_{\Lambda}\left\langle N^{+} \mid \Lambda\right\rangle^{J}\langle\Lambda| S_{\Lambda}^{J}\left(v^{+}=0, v^{\prime}=1|\Lambda\rangle\left\langle\Lambda \mid R^{\prime}\right\rangle^{J} .\right.
\end{aligned}
$$

Here, $R^{\prime}$ is the rotational quantum number of the $\mathrm{N}_{2}{ }^{+}$ion core of the $9 f$ Rydberg state. The central matrix element, $\langle\Lambda| S_{\Lambda}^{J}\left(v^{+}=0, v^{\prime}=1|\Lambda\rangle\right.$, is diagonal in $\Lambda$ (and $\lambda$ ), with the leading term proportional to the derivative of the corresponding quantum defect, $\mu_{\lambda}$, with respect to the internuclear distance.

Unfortunately, the quantum defect derivatives for the $f$ states were not determined in the detailed study of Jungen et al. $;{ }^{4}$ the values were small and assumed to be zero in their analysis. Although the $f \sigma$ and $f \pi$ components are known to be more penetrating and thus likely have bigger matrix

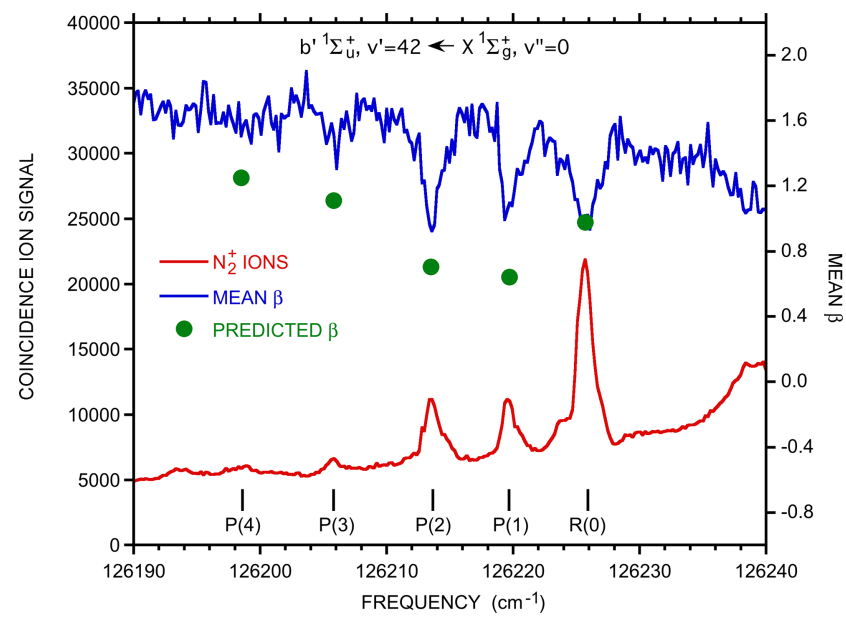

FIG. 3. Expanded portion of Fig. 1 in the region of the $b^{\prime 1} \Sigma_{\mathrm{u}}{ }^{+}, v^{\prime}=42$ band, along with the corresponding $\beta$ values. The theoretical $\beta$ values are the predicted values for the resonances corrected for the experimental direct photoionization continuum $\beta$ value, as discussed in the text.

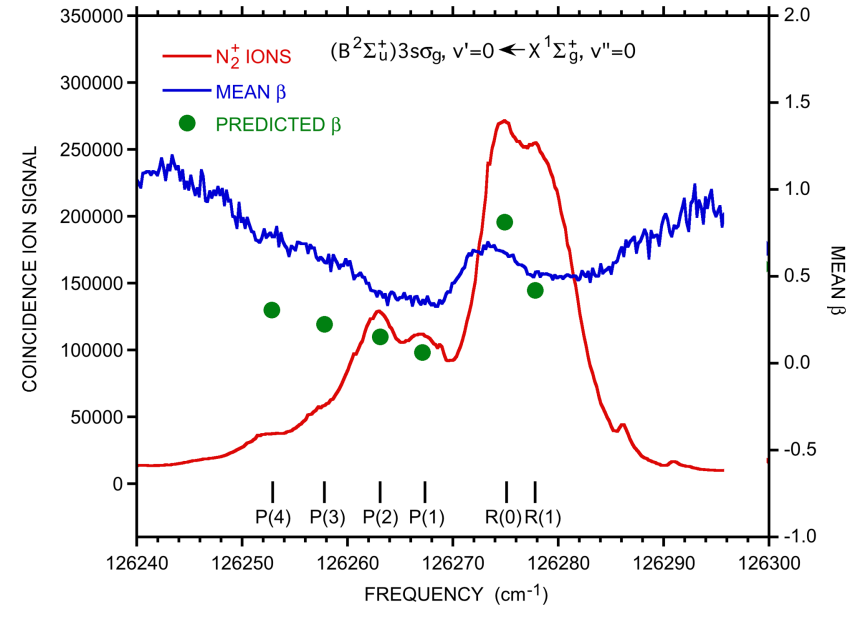

FIG. 4. Expanded portion of Fig. 1 in the region of the $\left(B^{2} \Sigma_{\mathrm{u}}{ }^{+}\right) 3 s \sigma_{g}$, $v^{\prime}=0$ band, along with the corresponding $\beta$ values. The theoretical $\beta$ values are the predicted values for the resonances corrected for the experimental direct photoionization continuum $\beta$ value, as discussed in the text.

elements than the higher $\lambda$ components, in the absence of detailed calculations, we assume these matrix elements to be independent of $\Lambda$ and $\lambda$. The middle factor in Eq. (10) is thus constant, and the sum over $\Lambda$ reduces the expression to $C\left\langle N^{+} \mid R^{\prime}\right\rangle=C \delta_{N^{+} R^{\prime}}$. That is, the rotational quantum number in the $9 f, v^{\prime}=1$ state is preserved upon vibrational autoionization. This result is surely approximate. ${ }^{38}$ While $N^{+}-R^{\prime}$ $=0$ transitions are expected to dominate in the vibrational autoionization process, some $N^{+}-R^{\prime}= \pm 2$ transitions will also occur as a result of the $\Lambda$ dependence of the matrix elements in Eq. (10).

\section{Comparison with experiment}

The experimental ion signal and $\beta$ values include contributions from both direct (nonresonant) photoionization and resonant autoionization, while our implementation of the theoretical expressions described above focuses only on the

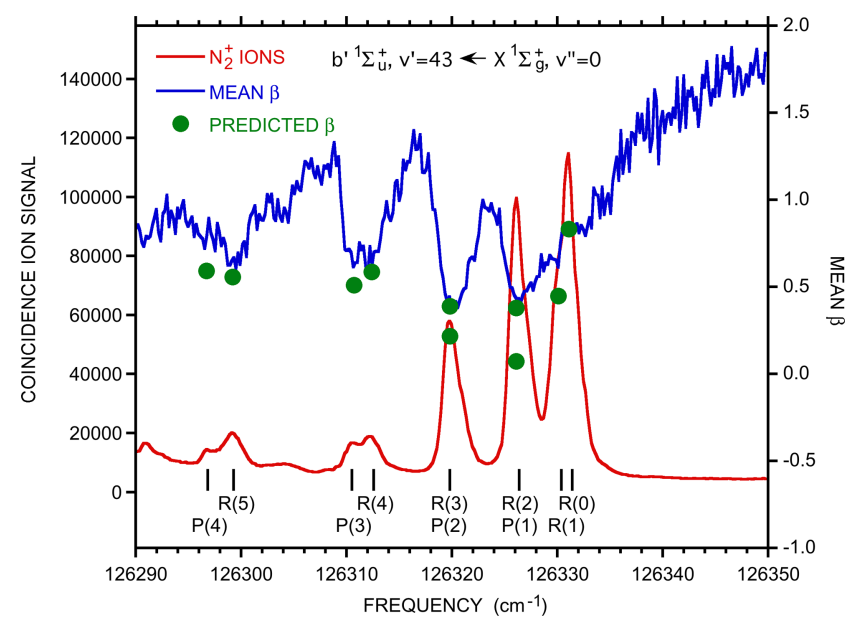

FIG. 5. Expanded portion of Fig. 1 in the region of the $b^{\prime}{ }^{1} \Sigma_{\mathrm{u}}{ }^{+}, v^{\prime}=43$ band, along with the corresponding $\beta$ values. The theoretical $\beta$ values are the predicted values for the resonances corrected for the experimental direct photoionization continuum $\beta$ value, as discussed in the text. 
resonant contribution. The nonresonant signal contains contributions from all $J^{\prime \prime}$ levels populated in the molecular beam, while the resonant process is for a single value of $J^{\prime \prime}$. As a result, a significant fraction of the nonresonant signal is incoherent with respect to the resonant signal. Thus, we make the approximation that the experimental $\beta$ value can be written as

$$
\beta_{\text {total }}=\frac{\beta_{\text {res }} I_{\text {res }}+\beta_{\text {cont }} I_{\text {cont }}}{\left(I_{\mathrm{res}}+I_{\text {cont }}\right)}
$$

or, equivalently,

$$
\beta_{\text {res }}=\frac{\beta_{\mathrm{tot}}\left(I_{\mathrm{res}}+I_{\mathrm{cont}}\right)-\beta_{\mathrm{cont}} I_{\mathrm{cont}}}{I_{\mathrm{res}}} .
$$

Here, $\beta_{\text {cont }}$ and $I_{\text {cont }}$ are the $\beta$ and intensity values for the continuum away from resonances. The nonresonant $\beta_{\text {cont }}$ values were determined from the average observed off-resonance values near the features of interest. The off-resonance $\beta_{\text {cont }}$

\begin{tabular}{|c|c|c|c|}
\hline $\begin{array}{l}\text { Transition } \\
\text { energy }\end{array}$ & Assignment & $\begin{array}{c}\text { Resonant } \beta \\
\text { theory }^{\mathrm{a}}\end{array}$ & $\begin{array}{c}\text { Resonant } \beta \\
\text { Expt. }^{\mathrm{b}}\end{array}$ \\
\hline 126160.40 & $X^{+} 6 p \sigma, v^{\prime}=2 P(2)$ & 0.100 & $0.24 \pm 0.07$ \\
\hline 126164.30 & $X^{+} 6 p \sigma, v^{\prime}=2 P(1)$ & 0.000 & $0.30 \pm 0.10$ \\
\hline 126173.00 & $X^{+} 6 p \sigma, v^{\prime}=2 R(0)$ & 0.800 & $0.80 \pm 0.07$ \\
\hline 126177.30 & $X^{+} 6 p \sigma, v^{\prime}=2 R(1)$ & 0.400 & $0.45 \pm 0.07$ \\
\hline 126183.10 & $X^{+} 6 p \sigma, v^{\prime}=2 R(2)$ & 0.320 & $0.36 \pm 0.18$ \\
\hline 126198.70 & $b^{\prime}, v^{\prime}=42 P(4)$ & 0.133 & $1.26 \pm 0.12$ \\
\hline 126205.80 & $b^{\prime}, v^{\prime}=42 P(3)$ & 0.114 & $1.07 \pm 0.16$ \\
\hline 126213.50 & $b^{\prime}, v^{\prime}=42 P(2)$ & 0.100 & $0.49 \pm 0.10$ \\
\hline 126219.60 & $b^{\prime}, v^{\prime}=42 P(1)$ & 0.000 & $0.67 \pm 0.05$ \\
\hline 126225.70 & $b^{\prime}, v^{\prime}=42 R(0)$ & 0.800 & $0.74 \pm 0.06$ \\
\hline 126252.60 & $B^{+} 3 s \sigma, v^{\prime}=0 P(4)$ & 0.133 & $0.57 \pm 0.09$ \\
\hline 126257.30 & $B^{+} 3 s \sigma, v^{\prime}=0 P(3)$ & 0.114 & $0.49 \pm 0.07$ \\
\hline 126262.90 & $B^{+} 3 s \sigma, v^{\prime}=0 P(2)$ & 0.100 & $0.38 \pm 0.11$ \\
\hline 126267.10 & $B^{+} 3 s \sigma, v^{\prime}=0 P(1)$ & 0.000 & $0.26 \pm 0.09$ \\
\hline 126274.80 & $B^{+} 3 s \sigma, v^{\prime}=0 R(0)$ & 0.800 & $0.59 \pm 0.09$ \\
\hline 126277.90 & $B^{+} 3 s \sigma, v^{\prime}=0 R(1)$ & 0.400 & $0.44 \pm 0.06$ \\
\hline 126296.80 & $b^{\prime}, v^{\prime}=43 P(4)$ & 0.133 & $0.35 \pm 0.09$ \\
\hline 126299.20 & $b^{\prime}, v^{\prime}=43 R(5)$ & 0.254 & $0.31 \pm 0.10$ \\
\hline 126310.70 & $b^{\prime}, v^{\prime}=43 P(3)$ & 0.114 & $0.23 \pm 0.12$ \\
\hline 126312.30 & $b^{\prime}, v^{\prime}=43 R(4)$ & 0.267 & $0.29 \pm 0.09$ \\
\hline 126319.80 & $b^{\prime}, v^{\prime}=43 P(2)$ & 0.100 & $0.21 \pm 0.14$ \\
\hline 126319.80 & $b^{\prime}, v^{\prime}=43 R(3)$ & 0.286 & $0.21 \pm 0.14$ \\
\hline 126326.10 & $b^{\prime}, v^{\prime}=43 P(1)$ & 0.000 & $0.29 \pm 0.10$ \\
\hline 126326.10 & $b^{\prime}, v^{\prime}=43 R(2)$ & 0.320 & $0.29 \pm 0.10$ \\
\hline 126330.20 & $b^{\prime}, v^{\prime}=43 R(1)$ & 0.400 & $0.54 \pm 0.11$ \\
\hline 126331.10 & $b^{\prime}, v^{\prime}=43 R(0)$ & 0.800 & $0.68 \pm 0.13$ \\
\hline 126469.80 & $X^{+} 9 f, v^{\prime}=1,{ }^{O} R(2)$ & 0.800 & 0.17 (ave) \\
\hline 126473.40 & $X^{+} 9 f, v^{\prime}=1, Q_{R(1)}$ & 0.800 & 0.36 (ave) \\
\hline 126480.00 & $X^{+} 9 f, v^{\prime}=1,{ }^{S} R(0)$ & 0.800 & 0.60 (ave) \\
\hline 126484.80 & $X^{+} 9 f, v^{\prime}=1, Q_{R(2)}$ & 0.250 & 0.79 (ave) \\
\hline 126492.70 & $X^{+} 9 f, v^{\prime}=1,{ }^{S} Q(1)$ & 0.500 & 0.29 (ave) \\
\hline
\end{tabular}

TABLE I. Angular distribution parameters for assigned $\mathrm{N}_{2}$ bands.

${ }^{\text {a Resonant } \beta \text { theory is the value determined directly by using Eqs. (4) and (5) or Eqs. (8) }}$ and (9) for the $X^{+} 9 f, v^{\prime}=1$ state.

${ }^{\mathrm{b}}$ As described in the text, resonant $\beta$ experiment is determined by subtracting the contribution from the nonresonant ionization processes from the experimental $\beta$ value. The values in the table correspond to the average of four measurements: two using the FWHM $\beta$ values and two using the full width at one-tenth maximum $\beta$ values. value drops significantly in the region of the $9 f, v^{\prime}=1$ states. This drop was also observed in the previous work of Holland et al. ${ }^{7}$ but the source of this decrease was not clear at that time.

Both expressions (11) and (12) are used to present the data. Figures 2-5 show expanded portions of Fig. 1 for the transitions to the $\left(X^{2} \Sigma_{\mathrm{g}}{ }^{+}\right) 6 p \sigma, v^{\prime}=2 ; b^{\prime}{ }^{1} \Sigma_{\mathrm{u}}{ }^{+}, v^{\prime}=42$; $\left(B^{2} \Sigma_{\mathrm{u}}{ }^{+}\right) 3 s \sigma_{g}, v^{\prime}=0$; and $b^{\prime}{ }^{1} \Sigma_{\mathrm{u}}{ }^{+}, v^{\prime}=43$ states, respectively. The experimental data are those from a single scan, and the $\beta$ values are those obtained by averaging between the onetenth-maximum values of the photoelectron peak. Rather than attempting to subtract the continuum contribution to all of the experimental $\beta$ values, the theoretical $\beta_{\text {res }}$ values have been used to determine theoretical $\beta_{\text {total }}$ values through Eq. (11), with the $I_{\text {res }}, I_{\text {cont }}$, and $\beta_{\text {cont }}$ values from the experimental spectrum. Because $\beta_{\text {cont }}$ is relatively large (we use $\beta_{\text {cont }}=1.73$ in Figs. $2-5$ ), the continuum contribution shifts the theoretical $\beta_{\text {res }}$ to higher values than the simple theory. Figures $2-5$ allow the visualization of the rotational dependence of the $\beta$ values, as well as the trends within the data.

Table I shows the averaged data from two experiments. Here we have used the experimental intensities and $\beta_{\text {total }}$ and $\beta_{\text {cont }}$ values with Eq. (12) to extract purely experimental $\beta_{\text {res }}$ values to compare directly with the theoretical $\beta_{\text {res }}$ values. For all but the $9 f$ state, the experimental $\beta$ values are the average of four values from two experiments, where we have used both the FWHM and FW at one-tenth maximum $\beta$ values. The error bars represent the standard deviations from the averaged values. For the $9 f$ data, the FWHM and FW at one-tenth maximum $\beta$ values from a single experiment are averaged.

\section{RESULTS AND DISCUSSION}

The off-resonant $\beta$ value in Figs. $1-5$ is approximately 1.73 and ranges from $\sim 1.6$ to 1.9 in the other data sets, although this off-resonance value falls to $\sim 1.2-1.3$ in the region of the $\left(X^{2} \Sigma_{\mathrm{g}}{ }^{+}\right) 9 f, v^{\prime}=1$ state. The off-resonance direct photoionization $\beta$ values observed between 126150 and $126450 \mathrm{~cm}^{-1}$ in Fig. 1 are reasonably consistent with the pure- $p$ value from Eq. (2) and the parameters from the work of Jungen et al. ${ }^{4}$ but are quite a bit higher than the corresponding value with the parameters from the work of Raoult et al. ${ }^{18}$ The present continuum $\beta$ values are also consistent with the values reported by $\mathrm{O}^{\prime}$ Keeffe et $a l .{ }^{6}$ and Holland et al., ${ }^{7}$ which were also determined using a jet-cooled sample. O'Keeffe et al. pointed out that their $\beta$ values are substantially larger than those observed by previous researchers using room-temperature samples and suggested that the difference might be due to the lower rotational quantum states populated in the cold sample. As discussed in Sec. III A, however, for direct photoionization into a pure $p$ continuum, Dill ${ }^{34}$ has shown that the finalrotational-state-averaged $\beta$ parameter is independent of the initial rotational quantum number. Of course, it is possible that the importance of the $f$ continuum grows with increasing rotational quantum number and lowers the overall $\beta$ value. Alternatively, some other explanation must account for the lower continuum $\beta$ values obtained in the earlier experimental studies. ${ }^{20,22,23}$ 
Figure 2 shows the comparison of the experimental and predicted $\beta$ values for individual rotational lines within the $\left(X^{2} \Sigma_{\mathrm{g}}{ }^{+}\right) 6 p \sigma, v^{\prime}=2$ band. Overall, the agreement is quite good. As seen in Table I, the extracted resonance contributions to the $\beta$ values are also in good agreement with the values extracted from the experiment. These observations suggest that the process is well described as vibrational autoionization of a $p \sigma$ state with the ejection of an $\ell=1$ photoelectron.

Figures 3 and 5 show the comparison of the experimental and predicted $\beta$ values for the bands corresponding to transitions to the $b^{\prime}{ }^{1} \Sigma_{\mathrm{u}}{ }^{+}, v^{\prime}=42$ and 43 states, respectively. As seen in Fig. 1, the $v^{\prime}=42$ band is quite weak, but the predicted value of $\beta$ does reproduce the experimental value for the strongest transition, as well as the trend for the weaker transitions. The subtraction of the nonresonant contribution to $\beta$ is more difficult when the peaks are very small compared to the continuum. Thus, the resonant contribution to $\beta$ extracted from the experiment in Table I does not agree very well with the predicted resonant contribution.

The transition to the $b^{\prime}{ }^{1} \Sigma_{\mathrm{u}}{ }^{+}, v^{\prime}=43$ state in Fig. 5 is significantly stronger than the one to the $v^{\prime}=42$ band, and here the agreement between the predicted and experimental $\beta$ values is quite good. The $R(2)$ and $P(1)$ lines, and the $R(3)$ and $P(2)$ lines are overlapped, and this figure shows the predicted values for both transitions. This figure suggests that the $R$-branch transitions are stronger than the $P$-branch transitions, but either way, the agreement - at least with respect to the trends—is reasonable. For the most part, this agreement also transfers over to the extraction of the resonant contributions to the observed $\beta$ values, as seen in Table I. Thus, for both the $b^{\prime}{ }^{1} \Sigma_{\mathrm{u}}{ }^{+}, v^{\prime}=42$ and 43 bands, it appears that the dominant contribution to the electronic autoionization mechanism involves a $p \sigma$ electron ejected as an $\ell=1$ photoelectron. However, the involvement of other $(\ell \neq 1)$ partial waves may account for the observed differences.

Figure 4 shows the comparison of the experimental and predicted $\beta$ values for the $\left(B^{2} \Sigma_{\mathrm{u}}{ }^{+}\right) 3 s \sigma_{g}$ Rydberg state. Here, the quantitative agreement between the experiment and predictions is not as good, although the theory does reproduce the overall trends in the experimental $\beta$ values. This behavior is also observed in Table I. These results suggest that electronic autoionization of the $\left(B \Sigma_{\mathrm{u}}{ }^{+}\right) 3 s \sigma_{g}$ state is more complicated than the simple ejection of an $\ell=1$ photoelectron. Sommavilla et $a .^{5}$ also identified the transition to the $\left(X^{2} \Sigma_{\mathrm{g}}{ }^{+}\right) 9 p \sigma, v^{\prime}=1$ state on the high-energy side of the features corresponding to transitions to the $\left(B^{2} \Sigma_{\mathrm{u}}^{+}\right) 3 s \sigma_{g}, v^{\prime}=0$ state. With the present resolution, the former band is buried under the much stronger and broader latter band, although the $R(1)$ transition can just be seen at $\sim 126286 \mathrm{~cm}^{-1}$. The background from the $\left(B^{2} \Sigma_{\mathrm{u}}{ }^{+}\right) 3 \mathrm{~s} \sigma_{g}, v^{\prime}=0$ resonances makes it difficult to extract isolated $\beta$ values for the $9 p \sigma, v^{\prime}=1$ band.

Figure 6 shows an expanded region of Fig. 1 that contains the unassigned band discussed in the Introduction. Actually, Sommavilla et al. ${ }^{5,16}$ have identified two bands in this region. In particular, with their extremely high resolution, they identified a number of sharp lines associated with the two small features observed between $\sim 126424$ and $126430 \mathrm{~cm}^{-1}$ in Fig. 6 that could be assigned to one band. The second band is associated with the much stronger, broader feature corresponding

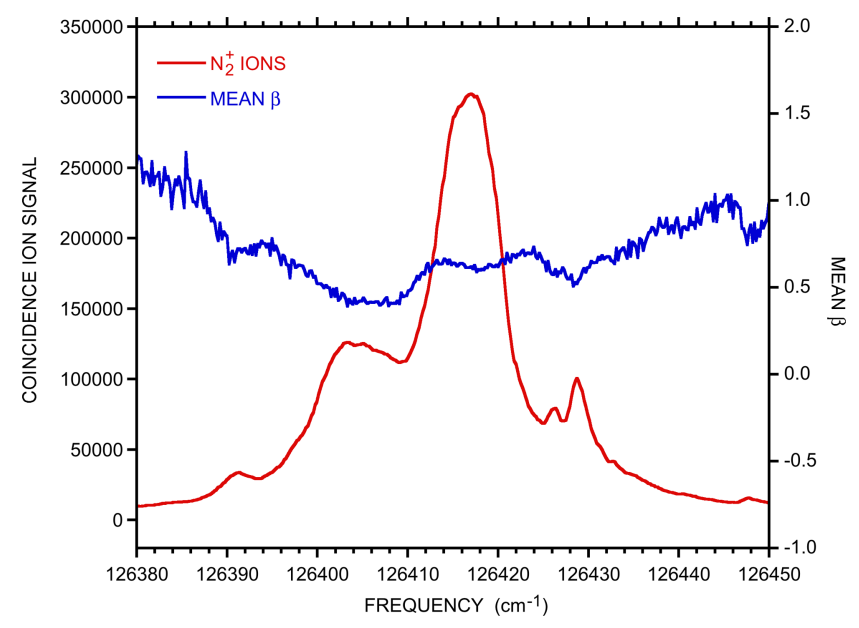

FIG. 6. Expanded portion of Fig. 1 in the region of the unassigned, intense feature, along with the corresponding $\beta$ values.

to the second cathedral tower. Without more detailed information, the $\beta$ values cannot provide significant help with the assignment. If we assume that either a pure ${ }^{1} \Sigma_{\mathrm{u}}{ }^{+}$or ${ }^{1} \Pi_{\mathrm{u}}$ state is autoionizing into the $X^{2} \Sigma_{\mathrm{g}}{ }^{+}, v^{+}=0, N^{+}+\varepsilon p \sigma$ or $\varepsilon p \pi$ continuum, respectively, Eqs. (4)-(7) can be used to predict the expected $\beta$ values. These values are given in Table II for the first few rotational levels of each branch for a ${ }^{1} \Sigma_{\mathrm{u}}{ }^{+}$or ${ }^{1} \Pi_{\mathrm{u}}$ autoionizing state. Unfortunately, the only substantial differences occur for the $R(0)$ transition, so it does not appear that the angular distributions can resolve this issue without a more detailed analysis.

Figure 7 shows an expanded region of Fig. 1 that includes the portion containing the $\left(X^{2} \Sigma_{\mathrm{g}}{ }^{+}\right) 9 f, v^{\prime}=1$ band. The assignments are based on the earlier work of McCormack et al. ${ }^{10,12}$ This band is very weak relative to most of the other bands, as seen in Fig. 1. In addition, the direct, nonresonant photoionization signal is stronger than in the lower energy region; thus, extracting the resonant contribution to the signal is more difficult than for the other bands. As mentioned above, the off-resonance continuum $\beta$ value is significantly smaller than it is in the lower energy portion of Fig. 1 , and the value $\beta$ $=1.19$ has been used to give the theoretical $\beta$ prediction shown in Fig. 7. The only major discrepancy between experiment and theory is for the $Q_{R(2)}$ transition. This

TABLE II. Theoretical angular distribution parameters for autoionizing ${ }^{1} \Sigma_{\mathrm{u}}{ }^{+}$ and ${ }^{1} \Pi_{\mathrm{u}}$ states.

\begin{tabular}{lcc}
\hline \hline Rotational transition & $\beta\left({ }^{1} \Sigma_{\mathrm{u}}{ }^{+}\right)$ & $\beta\left({ }^{1} \Pi_{\mathrm{u}}\right)$ \\
\hline$R(0)$ & 0.800 & 1.400 \\
$R(1)$ & 0.400 & 0.500 \\
$R(2)$ & 0.320 & 0.360 \\
$R(3)$ & 0.286 & 0.307 \\
\hline$Q(1)$ & & 0.500 \\
$Q(2)$ & & 0.700 \\
$Q(3)$ & & 0.750 \\
\hline$P(1)$ & 0.000 & \\
$P(2)$ & 0.100 & 0.150 \\
$P(3)$ & 0.114 & 0.143 \\
\hline \hline
\end{tabular}




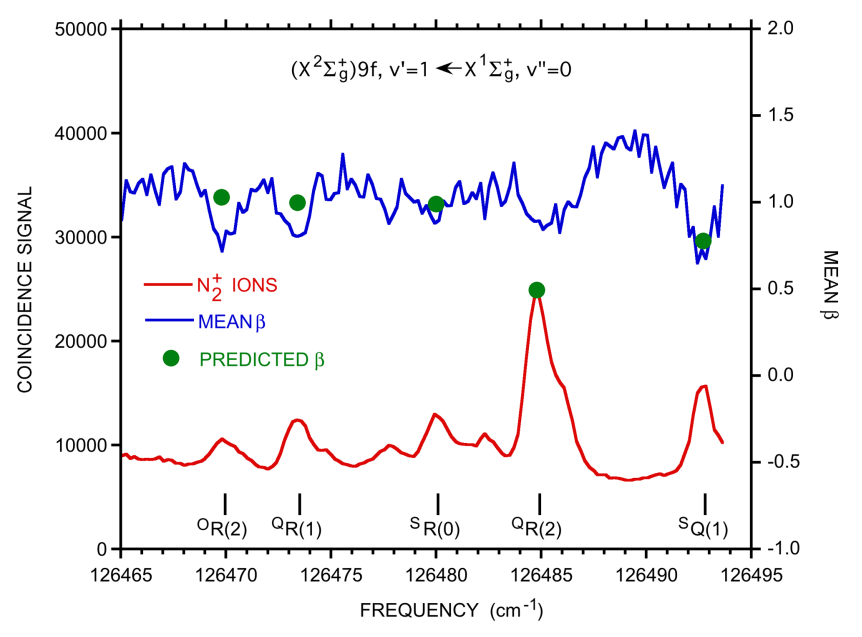

FIG. 7. Expanded portion of Fig. 1 in the region of the $\left(X^{2} \Sigma_{\mathrm{g}}{ }^{+}\right) 9 f, v^{\prime}=1$ band, along with the corresponding $\beta$ values. The theoretical $\beta$ values are the predicted values for the resonances corrected for the experimental direct photoionization continuum $\beta$ value, as discussed in the text.

transition is known to be perturbed by a transition to the $\left(A^{2} \Pi_{\mathrm{u}}\right) 3 d \pi^{1} \Delta_{\mathrm{u}}, v^{\prime}=2$ state, $^{12}$ which likely accounts for the significant discrepancy between experiment and theory. While the remaining continuum-corrected theoretical $\beta$ values in Fig. 7 are in reasonable agreement with the experiment, the same cannot be said of the on-resonance $\beta$ values shown in Table I. The difficulty here arises from the strong background with a relatively large $\beta$ value. Because the resonances are relatively weak, this situation results in a very small extracted value for the resonance $\beta$ value. Thus, it is difficult to determine if this disagreement between experiment and theory is a result of the weak signal or of the breakdown of the approximations used to predict the $\beta$ values for the $\left(X^{2} \Sigma_{\mathrm{g}}{ }^{+}\right) 9 f, v^{\prime}=1$ state. Theoretical calculations of the vibrational autoionization matrix elements and rotational branching ratios for this state would provide considerably more insight into the ionization dynamics.

\section{CONCLUSION}

We have presented a high-resolution photoionization spectrum in the near-threshold region of $\mathrm{N}_{2}$, along with the determination of the wavelength-dependent photoelectron angular distribution parameter. Rotational resolution in the excitation process allows a simplified theoretical description of the on-resonance $\beta$ values. In some instances, the simplified treatment provides quantitative agreement with the experiment. In other cases, the quantitative agreement is poor, although in most cases, the model still reproduces the qualitative behavior of the experimental measurements. This approach should be applicable to a significant number of small molecules. Nevertheless, the present approach is still relatively simplistic and ignores some of the subtleties associated with full MQDT calculations. For example, the earlier work on $\mathrm{H}_{2}$ has shown that the $\beta$ values can vary across the autoionizing resonances and may not reach a minimum (or maximum) at the corresponding minimum or maximum of the photoionization cross section. ${ }^{26}$ Furthermore, the present study accesses only a single vibrational level of the $\mathrm{N}_{2}{ }^{+} X^{2} \Sigma_{\mathrm{g}}{ }^{+}$ground state, and the $\beta$ values can also show a strong dependence on the vibrational final state. In spite of these deficiencies, the results do show that photoelectron angular distributions of rotationally resolved levels can provide insight into the character of the autoionizing resonance and the autoionization mechanism.

Molecular nitrogen, $\mathrm{N}_{2}$, is one of the most fundamental molecules and by far the dominant species in the Earth's atmosphere, where its interaction with VUV light is of significant importance. $\mathrm{N}_{2}$ has also played an important role in our understanding of strong-field ionization and high-harmonic generation, ${ }^{39-41}$ as well as in ground-breaking studies of attosecond dynamics. ${ }^{42}$ With these spectacular advances in ultrafast science, it is easy to forget that the near-threshold photoabsorption, photoionization, and photodissociation of $\mathrm{N}_{2}$ present extremely challenging problems in fundamental chemical dynamics, with the interaction of multiple valence states and Rydberg series converging to three different electronic states of $\mathrm{N}_{2}^{+}$. Significant features in this spectral region remain only tentatively assigned. O' Keeffe et al. ${ }^{6}$ have stressed the need for more detailed theoretical calculations throughout the near-threshold region in $\mathrm{N}_{2}$. In the present study, a simplified theoretical approach is used to predict the $\beta$ values for selected autoionizing resonances. While this approach is successful for some of the bands, we reiterate the need for a more detailed analysis of this prototypical molecular system.

\section{ACKNOWLEDGMENTS}

D.M.P.H. was supported by the Science and Technology Facilities Council, UK. This material is based on work supported by the U.S. Department of Energy, Office of Science, Office of Basic Energy Sciences, Division of Chemical Sciences, Geosciences, and Biosciences under Contract No. DE-AC02-06CH11357 (for S.T.P.). The experiments were performed on the DESIRS beamline at SOLEIL under Proposal Nos. 20140457 and 20150204. We are grateful to the entire staff of SOLEIL for running the facility.

${ }^{1}$ P. M. Dehmer, P. J. Miller, and W. A. Chupka, "Photoionization of $\mathrm{N}_{2} X$ ${ }^{1} \Sigma_{\mathrm{g}}{ }^{+}, v^{\prime \prime}=0$ and 1 near threshold. Preionization of the Worley-Jenkins Rydberg series," J. Chem. Phys. 80, 1030-1038 (1984).

${ }^{2}$ D. A. Shaw, D. M. P. Holland, M. A. MacDonald, A. Hopkirk, M. A. Hayes, and S. M. McSweeney, "A study of the absolute photoabsorption cross section and the photoionization quantum efficiency of nitrogen from the ionization threshold to $485 \AA$," Chem. Phys. 166, 379-391 (1992).

${ }^{3}$ K. P. Huber and Ch. Jungen, "High-resolution jet absorption study of nitrogen near 800 Å,” J. Chem. Phys. 92, 850-861 (1990).

${ }^{4}$ Ch. Jungen, K. P. Huber, M. Jungen, and G. Stark, "The near-threshold absorption spectrum of $\mathrm{N}_{2}$," J. Chem. Phys. 118, 4517-4538 (2003).

${ }^{5}$ M. Sommavilla, U. Hollenstein, G. M. Greetham, and F. Merkt, "Highresolution laser absorption spectroscopy in the extreme ultraviolet," J. Phys. B 35, 3901-3921 (2002).

${ }^{6}$ P. O'Keeffe, P. Bolognesi, A. Moise, R. Richter, Y. Ovcharenko, and L. Avaldi, "Vibrationally resolved photoionization of $\mathrm{N}_{2}$ near threshold," J. Chem. Phys. 136, 104307 (2012).

${ }^{7}$ D. M. P. Holland, E. A. Seddon, S. Daly, C. Alcaraz, C. Romanzin, L. Nahon, and G. A. Garcia, "The effect of autoionization on the $\mathrm{N}_{2}{ }^{+} \mathrm{X}^{2} \Sigma_{\mathrm{g}}{ }^{+}$state vibrationally resolved photoelectron anisotropy parameters and branching ratios," J. Phys. B 46, 095102 (2013). 
${ }^{8}$ R. Seiler, U. Hohenstein, G. M. Greetham, and F. Merkt, "Rydberg-stateresolved zero-kinetic-energy photoelectron spectroscopy," Chem. Phys. Lett. 346, 201-208 (2001)

${ }^{9}$ W. M. Kosman and S. Wallace, "Complete dipole oscillator strength distribution and its moments for $\mathrm{N}_{2}$," J. Chem. Phys. 82, 1385-1399 (1985).

${ }^{10}$ E. F. McCormack, S. T. Pratt, J. L. Dehmer, and P. M. Dehmer, "Doubleresonance spectroscopy of autoionizing states of $\mathrm{N}_{2}$ near the ionization threshold," Phys. Rev. A 42, 5445-5451 (1990).

${ }^{11}$ E. F. McCormack, S. T. Pratt, P. M. Dehmer, and J. L. Dehmer, "Doubleresonance spectroscopy of a complex resonance in molecular nitrogen," Phys. Rev. A 45, 4697-4705 (1992).

${ }^{12}$ E. F. McCormack, S. T. Pratt, J. L. Dehmer, and P. M. Dehmer, "Analysis of the $8 f, 9 f, 10 f, v=1$ Rydberg states of $\mathrm{N}_{2}$," Phys. Rev. A 44, 3007-3015 (1991).

${ }^{13}$ K. P. Huber, Ch. Jungen, K. Yoshino, K. Ito, and G. Stark, "The $f$ Rydberg series in the absorption spectrum of $\mathrm{N}_{2}$," J. Chem. Phys. 100, 7957-7972 (1994).

${ }^{14} \mathrm{H}$. Lefebvre-Brion, "Assignment in the near-threshold absorption spectrum of $\mathrm{N}_{2}$," J. Chem. Phys. 122, 144315 (2005).

${ }^{15}$ H. Lefebvre-Brion, "The "cathedral' in the near-threshold absorption spectrum of $\mathrm{N}_{2}$," J. Electron Spectrosc. Relat. Phenom. 144-147, 109-111 (2005).

${ }^{16} \mathrm{M}$. Sommavilla and F. Merkt, "On the rotational structure of a prominent band in the vacuum-ultraviolet spectrum of molecular nitrogen," J. Electron Spectrosc. Relat. Phenom. 151, 31-33 (2006).

${ }^{17}$ J. B. Randazzo, P. Croteau, O. Kostko, M. Ahmed, and K. A. Boering, "Isotope effects and spectroscopic assignments in the non-dissociative photoionization spectrum of $\mathrm{N}_{2}$," J. Chem. Phys. 140, 194303 (2014).

${ }^{18}$ M. Raoult, H. Le Rouzo, G. Raseev, and H. Lefebvre-Brion, "Ab initio approach to the multichannel quantum defect calculation of the electronic autoionisation in the Hopfield series of $\mathrm{N}_{2}$," J. Phys. B 16, 4601-4617 (1983).

${ }^{19}$ R. R. Lucchese, G. Raseev, and V. McKoy, "Studies of differential and total photoionization cross sections of molecular nitrogen," Phys. Rev. A 25, 2572-2587 (1982).

${ }^{20}$ S. H. Southworth, A. C. Parr, J. E. Hardis, and J. L. Dehmer, "Channel coupling and shape resonance effects in the photoelectron angular distributions of the $3 \sigma_{g}{ }^{-1}$ and $2 \sigma_{u}{ }^{-1}$ channels of $\mathrm{N}_{2}$," Phys. Rev. A 33, 1020-1023 (1986).

${ }^{21}$ B. Basden and R. R. Lucchese, "Vibrationally resolved cross sections and asymmetry parameters for the photoionization of $\mathrm{N}_{2}$ with coupling between the $\left(3 \sigma_{g}\right)^{-1}$ and the $\left(2 \sigma_{u}\right)^{-1}$ channels," Phys. Rev. A 37, 89-97 (1988).

${ }^{22}$ M. Zubek, G. C. King, and P. M. Rutter, "Near-threshold photoionisation studies of $\mathrm{N}_{2}$," J. Phys. B 21, 3585-3594 (1988).

${ }^{23}$ J. B. West, M. A. Hayes, A. C. Parr, J. E. Hardis, S. H. Southworth, T. A. Ferrett, J. L. Dehmer, X. M. Hu, and G. V. Marr, "A high-resolution angle resolved photoelectron-spectroscopy study of $\mathrm{N}_{2}$," Phys. Scripta 41, 487-489 (1990)

${ }^{24}$ K. Ueda, J. B. West, M. A. Hayes, M. R. F. Siggel, A. C. Parr, and J. L. Dehmer, "Photoelectron study of electronic autoionization in rotationally cooled $\mathrm{N}_{2}$ : The $n=6$ member of the Hopfield series," J. Phys. B 26, L601-L606 (1993)
${ }^{25}$ D. M. P. Holland and D. A. Shaw, "The effect of autoionising Rydberg states on the formation of low energy electrons," J. Electron Spectrosc. Relat. Phenom. 184, 144-148 (2011).

${ }^{26} \mathrm{M}$. Raoult, Ch. Jungen, and D. Dill, "Photoelectron angular distributions in $\mathrm{H}_{2}$ : Calculation of rotational-vibrational preionization by multichannel quantum defect theory," J. Chim. Phys. 77, 599-604 (1980).

${ }^{27}$ G. Öhrwall, P. Baltzer, and J. Bozek, "Rotational branching ratios in $\mathrm{N}_{2}{ }^{+}$: Observation of photon energy dependence by photoelectron spectroscopy," Phys. Rev. Lett. 81, 546-549 (1998).

${ }^{28} \mathrm{G}$. Öhrwall, P. Baltzer, and J. Bozek, "Photoelectron spectra of $\mathrm{N}_{2}{ }^{+}$: Rotational line profiles studied with He I-excited angle-resolved spectroscopy and with synchrotron radiation,” Phys. Rev. A 59, 1903-1912 (1999).

${ }^{29}$ H. Park, I. Konen, and R. N. Zare, "Partial-wave decomposition of the ionization continuum accessed by vibrational autoionization of the $\mathrm{NO}(v$ $=1, N=20, N_{R}{ }^{+}=20$ ) level," Phys. Rev. Lett. 84, 3819-3822 (2000).

${ }^{30}$ K. L. Reid, "Photoelectron angular distributions," Annu. Rev. Phys. Chem. 54, 397-424 (2003).

${ }^{31}$ L. Nahon, N. de Oliveira, G. A. Garcia, J. F. Gil, B. Pilette, O. Marcouillé, B. Lagarde, and F. Polack, "DESIRS: A state-of-the-art VUV beamline featuring high resolution and variable polarization for spectroscopy and dichroism at SOLEIL,” J. Synchrotron Radiat. 19, 508-520 (2012).

${ }^{32}$ G. A. Garcia, B. K. Cunha de Miranda, M. Tia, S. Daly, and L. Nahon, "DELICIOUS III: A multipurpose double imaging particle coincidence spectrometer for gas phase vacuum ultraviolet photodynamics studies," Rev. Sci. Instrum. 84, 053112 (2013).

${ }^{33}$ X. Tang, G. A. Garcia, J.-F. Gil, and L. Nahon, "Vacuum upgrade and enhanced performances of the double imaging electron/ion coincidence endstation at the vacuum ultraviolet beamline DESIRS," Rev. Sci. Instrum. 86, 123108 (2015).

${ }^{34}$ D. Dill, "Angular distributions of photoelectrons from $\mathrm{H}_{2}$ : Effects of rotational autoionization," Phys. Rev. A 6, 160-172 (1972).

${ }^{35}$ U. Fano and D. Dill, "Angular momentum transfer in the theory of angular distributions," Phys. Rev. A 6, 185-192 (1972).

${ }^{36}$ G. A. Garcia, L. Nahon, and I. Powis, "Two-dimensional charged particle image inversion using a polar basis function expansion,” Rev. Sci. Instrum. 75, 4989-4996 (2004).

${ }^{37}$ H. H. Michels, "Electronic structure of excited states of selected atmospheric systems," Adv. Chem. Phys. 45, 225-340 (1981).

${ }^{38}$ E. E. Eyler, "Autoionization of nonpenetrating Rydberg states in diatomic molecules," Phys. Rev. A 34, 2881-2888 (1986).

${ }^{39}$ J. Itatani, J. Levesque, D. Zeidler, H. Niikura, H. Pepin, J. C. Kieffer, P. B. Corkum, and D. M. Villeneuve, "Tomographic imaging of molecular orbitals," Nature 432, 867-871 (2004).

${ }^{40}$ A.-T. Le, R. R. Lucchese, and C. D. Lin, "Uncovering multiple orbitals influence in high harmonic generation from aligned $\mathrm{N}_{2}$," J. Phys. B 42, 211001 (2009).

${ }^{41}$ Y. Mairesse, J. Higuet, N. Dudovich, D. Shafir, B. Fabre, E. Mével, E. Constant, S. Patchkovskii, Z. Walters, M. Yu. Ivanov, and O. Smirnova, "High harmonic spectroscopy of multichannel dynamics in strong-field ionization," Phys. Rev. Lett. 104, 213601 (2010).

${ }^{42}$ W. Cao, E. R. Warrick, A. Fidler, D. M. Neumark, and S. R. Leone, "Noncollinear wave mixing of attosecond XUV and few-cycle optical laser pulses in gas-phase atoms: Toward multidimensional spectroscopy involving XUV excitations," Phys. Rev. A 94, 053846 (2016). 